\title{
Quand les bactéries modulent leur vitesse d'évolution selon l'environnement
}

\begin{abstract}
L'évolution darwinienne est la sélection du variant le plus adapté produit par des phénomènes de mutation et/ou de recombinaison $(\mathrm{m} / \mathrm{s}$ 1997, $\left.n^{\circ} 3, p . I V\right)$. La contribution de chacun de ces deux mécanismes à la production de la diversité génétique dépend des espèces. Il a été estimé que chez Escherichia coli, malgré la structure clonale de ses populations naturelles [1], chaque variation nucléotidique a 50 fois plus de chance d'être apparue par recombinaison (avec un partenaire portant ce changement nucléotidique) que par une mutation de novo [2]. Le système de réparation des mésappariements (SRM) joue un rôle clé dans le contrôle de la stabilité (anti-variabilité) du génome en agissant, comme nous le verrons plus loin, sur ces deux phénomènes, mutation et recombinaison [3].
\end{abstract}

Des études du taux de mutations dans des populations naturelles de bactéries ont montré qu'il existe une proportion non négligeable (de 1 à $10 \%$ ) de souches ayant un taux de mutation élevé (bactéries mutatrices ou mutateurs), ceci étant dû essentiellement à une inactivation de leur SRM [4-6]. Une telle fréquence de mutateurs parmi les isolats naturels suggère la présence de conditions sélectionnant un taux de mutation élevé dans la nature. Il a été montré expérimentalement que la fraction de mutateurs parmi des $E$. coli peut augmenter quand la population est soumise à une sélection très forte (traitement antibiotique) [7] ou prolongée (adaptation à un nouvel environnement) [8]. Les mutateurs sont alors sélectionnés par leur association avec les rares mutations favorables qu'ils produisent, et ce malgré le coût des très nombreuses mutations délétères produites [9]. Quand l'adaptation est achevée, le coût des tionne un taux de mutation élevé. La production continue de mutations délétères par des cellules mutatrices conduit à une rapide érosion du génome quand les populations passent par de sévères goulets d'étranglement [10]. Les allèles adaptatifs peuvent être sauvés s'ils sont transférés horizontalement à des bactéries non mutatrices ou si le taux de mutations des souches mutatrices ayant acquis la (ou les) mutation(s) adaptative(s) est réduit. Cette réduction du taux de mutation peut être obtenue par plusieurs mécanismes: une réversion de la mutation ayant entraîné le phénotype mutateur, une acquisition de mutations suppressives ou la réacquisition du gène à fonction antimutatrice par transfert horizontal. Nous avons testé cette dernière hypothèse en confrontant les données de l'analyse de mutants du SRM obtenus au laboratoire et les données de séquences des gènes du SRM d'isolats naturels [11].

\section{Le système de réparation} des mésappariements (SRM)

Le SRM a un rôle capital dans le contrôle de la fidélité de la réplication de l'ADN. Quatre gènes sont nécessaires pour une fonction normale du SRM chez E. coli: mutS, mutL, mutH et mutU (uvrD) [12]. Un homodimère de la protéine MutS reconnaît et se fixe sur les nucléotides non appariés ou mal appariés dans la molécule d'ADN. La protéine MutL, également en homodimère, s'associe au complexe MutS/ADN. Le complexe protéique va alors migrer à la recherche d'un site 5'GATC-3' hémiméthylé et recruter MutH. La méthylation de l'adénine par la méthylase Dam ne se produit en effet que quelques minutes après la réplication, et les sites GATC sont donc hémiméthylés en période post- réplicative. Il y aura ensuite activation de MutH qui va cliver et exciser le brin non méthylé qui correspond à celui qui vient d'être synthétisé avec l'erreur reconnue par MutS. Cette étape sera suivie de la synthèse du brin d'ADN. L'hélicase II (MutU) permet le déroulement de l'ADN lors de ces deux dernières étapes. L'inactivation de n'importe lequel des 4 gènes du SRM augmente les taux de mutations ponctuelles de 100 à 1000 fois par rapport à une bactérie sauvage.

Le mésappariement des bases dans l'ADN peut aussi survenir durant la recombinaison entre séquences d'ADN non identiques. Le SRM reconnaît de telles structures et agit comme un inhibiteur de la recombinaison entre des séquences d'ADN divergentes. Cette fonction est essentielle pour la maintenance de l'intégrité structurale des chromosomes en empêchant les duplications et conversions géniques entre séquences homologues. Le SRM contrôle aussi la recombinaison post-conjugaison et post-transduction entre de l'ADN de souches ou d'espèces différentes [13, 14]. Toutefois, alors que l'inactivation de n'importe quel gène du SRM augmente la fréquence de mutations ponctuelles de la même façon, l'inactivation de différents gènes du SRM a des effets distincts et caractéristiques sur la recombinaison entre souches différentes [11]. L'augmentation du taux de recombinaison (effet " hyperrecombinant») est très importante si les gènes mutS et mutL sont inactivés, et plus modeste s'il s'agit des gènes mutH et mutU. La présence de cassures simple brin de l'ADN dans les phénomènes de recombinaison peut expliquer l'effet modéré de l'inactivation de mutH (l'activité d'hémiendonucléase de MutH n'est plus nécessaire) tandis que MutU est probablement remplacée par d'autres 
enzymes impliquées dans la recombinaison tel RecG.

\section{Transfert horizontal des gènes du SRM}

La fréquence de transferts horizontaux de gènes détectables dans les populations naturelles dépend du taux de transfert de la cellule donatrice vers la receveuse, du taux de recombinaison et de la valeur adapta- tive des recombinants. Les gènes "de ménage " sont peu échangés à l'inverse des gènes codant pour des antigènes de surface ou des cibles de résistance aux antibiotiques dont la variabilité amène un avantage sélectif immédiat à la bactérie exposée au système immunitaire ou aux antibiotiques.

Nous avons quantifié le taux de transfert horizontal des gènes SRM dans des isolats naturels d'E. coli. Les trans- ferts de gènes chromosomiques entre les souches peuvent être mis en évidence lorsqu'il existe une incongruence (absence de concordance) entre la phylogénie du gène et la phylogénie des souches $[15,16]$. La phylogénie des souches d'E. coli, considérée comme la «vraie phylogénie» de l'espèce, est fondée sur l'étude de nombreux locus par électrophorèse d'enzymes métaboliques, les polymorphismes de restriction de
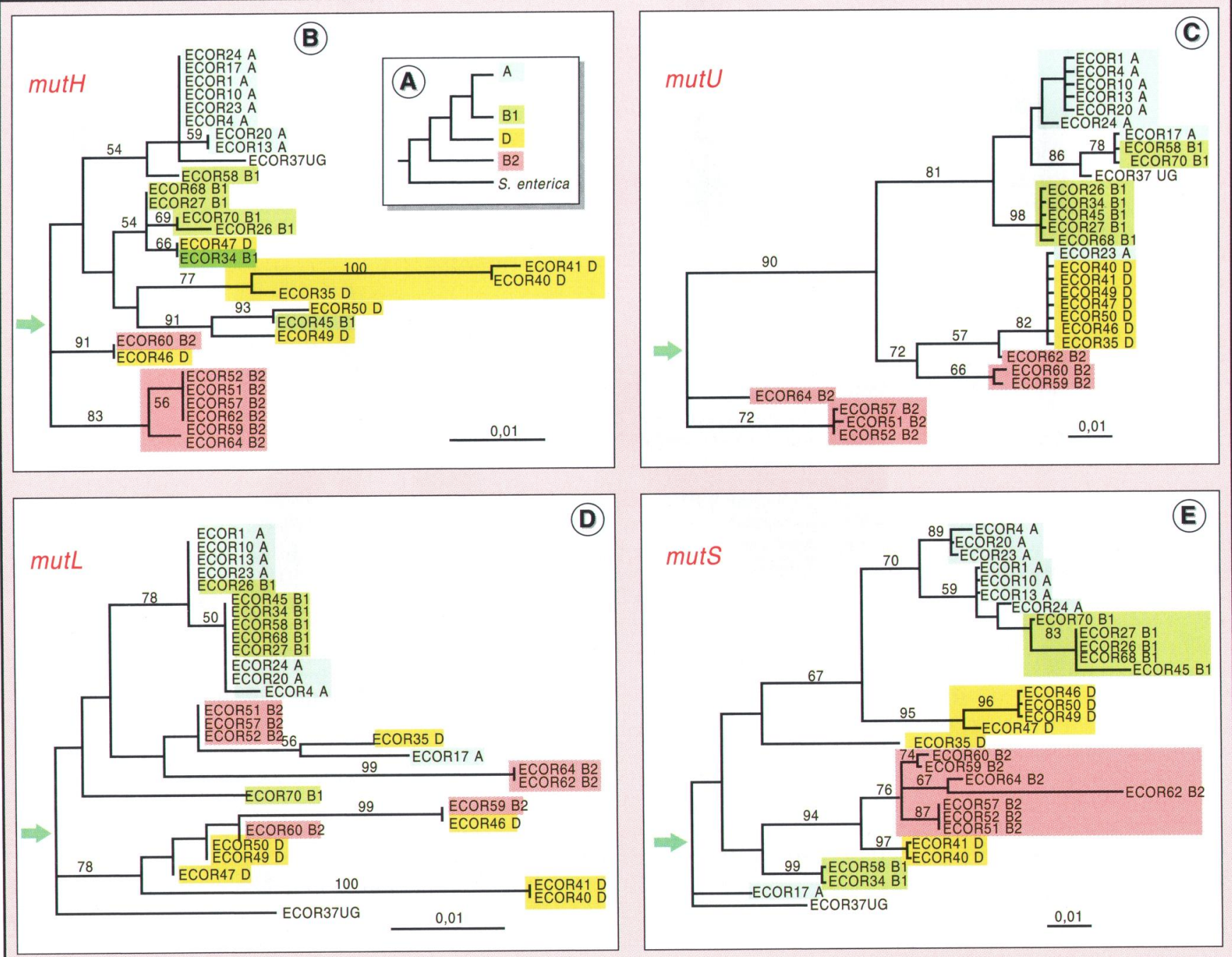

Figure 1. Arbres phylogénétiques des gènes du SRM chez 30 isolats naturels $d$ E. coli appartenant à la collection ECOR [18]. Quatre groupes phylogénétiques ont été identifiés chez E. coli $(A, B 1, D, B 2)$ et quelques souches n'appartiennent à aucun de ces groupes (UG = ungrouped). A. Phylogénie des souches (d'après [1, 17]). B à E. Phylogénie des gènes du SRM. Les arbres ont été obtenus par la méthode du maximum de vraisemblance. Les flèches montrent le point de branchement du groupe extérieur (racine de l'arbre) qui est Haemophilus influenzae pour mutU et S. enterica pour mutH, mutL et mutS. Les nombres en dessous des branches correspondent aux valeurs de bootstrap $\geq 50$ (100 réplications). Les barres d'échelle indiquent le nombre de substitutions de nucléotide par site. Noter dans les différentes phylogénies des gènes que les souches d'un même groupe (même couleur) ne se retrouvent pas ensemble, indiquant la présence d'événements de recombinaison par transfert horizontal.

$m / s n^{\circ} 4$, vol. 17 , avril 2001 
l'ADN ribosomique, l'amplification au hasard et la séquence de gènes métaboliques [1, 17]. La phylogénie des 4 gènes du SRM sur un échantillon de 30 isolats naturels appartenant aux différents groupes phylogénétiques d'E. coli (A, B1, D et B2) montre clairement que, pour ces gènes, on ne retrouve pas la phylogénie d'espèce car les souches d'un même groupe phylogénétique ne sont pas regroupées ensemble (figure 1). Ceci indique l'existence de transferts horizontaux. A l'aide d'un logiciel informatique que nous avons développé, nous avons quantifié sur les séquences d'ADN des gènes de ménage et des gènes du SRM la présence de transfert de l'ADN entre souches de groupes phylogénétiques différents (mosaïcisme). Tout d'abord, les différents gènes du SRM n'ont pas le même degré de recombinaison intragénique: mutS surtout et mutL ont un niveau de mosaïcisme supérieur à celui de mutU et mutH. Ensuite, tous les gènes du SRM ont de manière significative un degré de recombinaison supérieur à celui des gènes de ménage. Ainsi, au cours de l'évolution, les gènes du SRM, et surtout mutS, ont fréquemment subi des événements de recombinaison intragénique.

\section{Un scénario évolutif}

Différents gènes du SRM ont dans leurs séquences des traces de recombinaison variables: $m u t S$ et $m u t L$ ont un plus haut degré de mosaïcisme que mutU et mutH. De même, l'inactivation de $m u t S$ et de $m u t L$ entraîne un phénotype «hyper-recombinant» beaucoup plus important que l'inactivation de mutU et mutH. Cette corrélation hautement significative entre ces deux variables suggère fortement que les événements de recombinaison inférés à partir de la structure mosaïque des gènes du SRM est survenue dans des moments où la bactérie avait inactivé son SRM. Notre travail suggère que les fonctions du SRM ont été perdues et réacquises de manière répétitive durant l'histoire évolutive d'E. coli, et que la restauration des fonctions antimutatrices survient par échange géné- types d'hypermutation et d'hyperrecombinaison permet la génération rapide de diversité génétique sur laquelle la sélection va jouer durant l'adaptation et, en même temps, offre un moyen de restaurer un taux de mutation normal après l'adaptation. Ainsi, la proportion variable de mutateurs ayant inactivé leur SRM pourrait représenter une sorte de "changement de vitesse " capable de moduler la vitesse d'évolution des populations bactériennes.

\section{Remerciements}

Ce travail a été subventionné en partie par le Programme de recherche fondamentale en microbiologie et maladies infectieuses et paramicrobiologie et maladies infectieuses et para-
sitaires, ministère de l'Éducation nationale, de la Recherche et de la Technologie, et par le Programme environnement et santé - ministère de l'Aménagement du Territoire et de l'Environnement.

1. Desjardins P, Picard B, Kaltenböck B, Elion J Denamur E. Sex in Escherichia coli does not disrupt the clonal structure of the population: evidence from random amplified polymorphic DNA and restriction-fragment length polymorphism. I Mol Evol 1995; 41: 440-8.

2. Guttman DS, Dykhuizen DE. Clonal divergence in Escherichia coli as a result of recombination, not mutation. Science 1994; 266: 1380-3.

3. Radman M, Matic I, Halliday JA, Taddei F. Editing DNA replication and recombinaison by mismatch repair: from bacterial genetics to mechanisms of predisposition to cancer in humans. Philos Trans R Soc Lond B Biol Sci 1995; 347: 97 103.

4. LeClerc JE, Baouguang L, Payne WL, Cebula TA. High mutation frequencies among Escherichia coli and Salmonella pathogens. Science 1996; 274: 1208-11.

5. Matic I, Radman M, Taddei F, et al. Highly variable mutation rates in commensal and pathogenic Escherichia coli. Science 1997; 277 : 1833-4.

6. Oliver A, Canton R, Campo P, Baquero F, Blazquez J. High frequency of hypermutable Pseudomonas aeruginosa in cystic fibrosis lung infection. Science 2000; 288: 1251-4.

7. Mao EF, Lane L, Lee J, Miller JH. Proliferation of mutators in a cell population. J Bacteriol 1997; 179: 417-2.

8. Sniegowski PD, Gerrish PJ, Lenski RE. Evolution of high mutation rates in experimental populations of E. coli. Nature 1997; 387: 703-5.

9. Taddei F, Radman M, Maynard-Smith J, Toupance B, Gouyon PH, Godelle B. Role of mutator alleles in adaptive evolution. Nature 1997; 387 : 700-2.

10. Funchain P, Yeung A, Stewart JL, Lin R, Slupska MM, Miller JH. The consequences of growth of a mutator strain of Escherichia coli as measured by loss of function among multiple gene targets and loss of fitness. Genetics 2000 154: 959-70.

11. Denamur E, Lecointre G, Darlu P, et al. Evolutionary implications of the frequent horizontal transfer of mismatch repair genes. Cell 2000; 103: 711-21.

12. Friedberg EC, Walker GC, Siede W. DNA repair and mutagenesis. Washington DC: ASM Press, 1995.

13. Rayssiguier C, Thaler DS, Radman M. The barrier to recombination between Escherichia col and Salmonella typhimurium is disrupted in mismatch-repair mutants. Nature 1989 ; 342 : 396-401. 14. Matic I, Rayssiguier C, Radman M. Interspecies gene exchange in bacteria: the role of SOS and mismatch repair systems in evolution of species. Cell 1995; 80: 507-15.

15. Dykhuizen DE, Green L. Recombination in Escherichia coli and the definition of biological species. J Bacteriol 1991; 173: 7257-68.

16. Bull JJ, Huelsenbeck JP, Cunningham CW, Swofford DL, Waddel PJ. Partitioning and combining data in phylogenetic analysis. Syst Biol 1993; 42: 384-97.

17. Lecointre G, Rachdi L, Darlu P, Denamur E Escherichia coli molecular phylogeny using the incongruence length difference test. Mol Biol Evol $1998 ; 15: 1685-95$.

18. Ochman H, Selander RK. Standard reference strains of Escherichia coli from natural populations. J Bacteriol 1984; 157: 690-3.

\section{Erick Denamur Latif Rachdi}

Inserm U. 458, Hôpital Robert-Debré, 48, boulevard Sérurier, 75019 Paris, France.

\section{Guillaume Lecointre}

Muséum national d'histoire naturelle, 43, rue Cuvier, 75005 Paris, France.

\section{Pierre Darlu}

Inserm U. 535, Hôpital Kremlin-Bicêtre, 80, rue du Général-Leclerc, 94276 Le Kremlin-Bicêtre, France.

\section{Olivier Tenaillon}

François Taddei

Miroslav Radman

Ivan Matic

Inserm E. 9916, Faculté de médecine Necker-Enfants Malades, Paris, France. 